\title{
Modified Appleby Procedure with Arterial Reconstruction for Locally Advanced Pancreatic Adenocarcinoma: A Literature Review and Report of Three Unusual Cases.
}

\author{
Jessica A. Latona \\ Thomas Jefferson University \\ Kathleen M. Lamb \\ Thomas Jefferson University \\ Michael J. Pucci \\ Thomas Jefferson University \\ Warren R. Maley

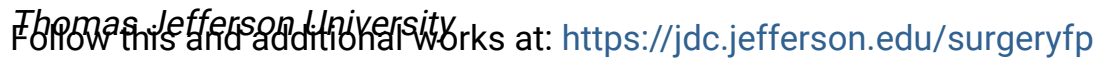 \\ arbesof.tMeGrastroenterology Commons, and the Surgery Commons \\ Theras is feferso Uwiversitw access to this document benefits you
}

\author{
Recommended Citation \\ Latona, Jessica A.; Lamb, Kathleen M.; Pucci, Michael J.; Maley, Warren R.; and Yeo, Charles J., \\ "Modified Appleby Procedure with Arterial Reconstruction for Locally Advanced Pancreatic \\ Adenocarcinoma: A Literature Review and Report of Three Unusual Cases." (2016). Department \\ of Surgery Faculty Papers. Paper 136. \\ https://jdc.jefferson.edu/surgeryfp/136
}

This Article is brought to you for free and open access by the Jefferson Digital Commons. The Jefferson Digital Commons is a service of Thomas Jefferson University's Center for Teaching and Learning (CTL). The Commons is a showcase for Jefferson books and journals, peer-reviewed scholarly publications, unique historical collections from the University archives, and teaching tools. The Jefferson Digital Commons allows researchers and interested readers anywhere in the world to learn about and keep up to date with Jefferson scholarship. This article has been accepted for inclusion in Department of Surgery Faculty Papers by an authorized administrator of the Jefferson Digital Commons. For more information, please contact: JeffersonDigitalCommons@jefferson.edu. 


\title{
Modified Appleby Procedure with Arterial Reconstruction:
}

\author{
A Literature Review and Report of 3 Unusual Cases \\ Jessica A Latona MD, Kathleen M Lamb MD, Daniel M Relles MD, Warren Maley MD, and \\ Charles J Yeo MD, FACS
}

Department of Surgery, Thomas Jefferson University Hospital

The Jefferson Pancreas, Biliary and Related Cancer Center

\author{
Philadelphia, PA
}

Presented as a poster at the 2015 Society for Surgery of the Alimentary Tract and the 2015

Pancreas Club; Washington, DC: May 2015

Corresponding Author:

Charles J. Yeo, MD, FACS

Samuel D. Gross Professor and Chair of Surgery

Thomas Jefferson University

1015 Walnut Street

620 Curtis Building

Philadelphia, Pennsylvania 19107

Phone: 215-955-8643

Fax: 215-923-6609

Charles.Yeo@jefferson.edu

Running head: Appleby with Arterial Reconstruction 


\begin{abstract}
BACKGROUND: Pancreatic body and tail carcinomas are often diagnosed with local vascular invasion of the celiac axis (CA) and its branches. With such involvement, these tumors have traditionally been considered unresectable. The modified Appleby procedure allows margin negative resection of some such locally advanced tumors. This procedure involves distal pancreatectomy with en bloc splenectomy and CA resection and relies on the presence of collateral arterial circulation via an intact pancreaticoduodenal arcade and the gastroduodenal artery (GDA). When the resultant collateral circulation is inadequate to provide sufficient hepatic and gastric arterial inflow, arterial reconstruction (AR) is necessary to "supercharge" the inflow. Herein, we review all reported cases of AR with modified Appleby procedures that we have discovered in the literature, and we report our experience of 3 recent cases with arterial reconstruction including 2 cases with arterial bypasses not requiring interposition grafting.
\end{abstract} METHODS: A PubMed search was systematically completed of studies relating to distal pancreatectomy with CA resection and subsequent AR.

RESULTS: Eleven reports involving 27 patients were identified of distal and total pancreatectomy with AR after CA resection. The most common AR in the literature, performed in 11 patients, was a bypass from the aorta to the common hepatic artery (CHA) using a variety of interposition conduits. In our experience, patient \#1 had a primary side to end aorto-CHA bypass, patient \#2 had a primary end to end bypass of the distal CHA to the left gastric artery in the setting a replaced left hepatic artery, and patient \#3 required an aortic to proper hepatic artery bypass with saphenous vein graft and portal venous reconstruction. Patient \#1 received adjuvant chemo-radiation therapy. Patients \#2 and \#3 received neoadjuvant chemotherapy and adjuvant chemotherapy for postoperative metastatic disease. All patients recovered from their operations 
without ischemic complications and they are currently 11, 10 and 8 months post-op, respectively. CONCLUSIONS: The criteria for resectablilty in patients with locally advanced pancreatic body and tail neoplasms are expanding due to increasing experience with AR in the setting of the modified Appleby procedure. When performing AR, primary anastomosis may be considered preferable to interposition grafting as it decreases the potential for the infectious and thrombotic complications associated with conduits and it reduces the number of vascular anastomoses from two to one. Consideration must also be given to normal variant anatomy of the hepatic circulation (as seen in patient \#2) during operative planning as the LGA is resected with the CA. The modified Appleby with AR, when used in appropriately selected patients, offers the potential for safe, margin negative resection of locally advanced tumors.

Key words: celiac axis; pancreatic cancer; resection; Appleby procedure 


\section{INTRODUCTION}

Pancreatic body and tail carcinomas account for approximately one-third of pancreatic neoplasms. They are frequently identified at a late stage compared to pancreatic head neoplasms, as symptoms are not manifested until there is local invasion of major vessels and associated neural plexuses. Consequently, metastases, retroperitoneal invasion, or involvement of major vessels (such as the common hepatic artery and splenic artery) have traditionally rendered these tumors to be unresectable. Unresected patients have a clinical decline suffering from back pain from local nerve invasion, ileus related to peritoneal dissemination and ultimately a median survival of only 6 months. In the last decade improved computerized tomographic (CT) imaging protocols to visualize local invasion and an aggressive surgical approach via the modified Appleby procedure has allowed some traditionally unresectable lesions to be resected, improving patient outcomes and palliating pain.

The Appleby operation, en bloc resection of the celiac axis, distal pancreatectomy and total gastrectomy, was first performed in 1953 in order to achieve a more complete resection of celiac axis lymph nodes in locally advanced gastric cancer (Appleby 1953). Nimura adopted this

procedure for the resection of a pancreatic body and tail carcinoma in 1976 (Nimura 1976). Since then, extended resection and radical lymphadenectomy has been widely practiced by Japanese surgeons (Wu et al), while it's adoption in Western medicine have been delayed. In 2005, Makary and colleagues became the first American group to report their experience with an Appleby procedure in a 72 year-old male with a pancreatic body cancer invading the posterior stomach (Makary 2005). [Not sure this is true; see Fortner's work from the 1970s.] Subsequently, the Appleby procedure was modified for use in pancreatic cancer to include a distal pancreatectomy with en bloc splenectomy and celiac axis resection with ligation of the 
celiac artery at its aortic takeoff, extirpation of the common hepatic artery (CHA) proximal to the gastrodouodenal artery (GDA), and left gastric artery (LGA) without gastric resection. The feasibility of the procedure is based on the presence of collateral circulation between the superior mesenteric artery (SMA) and the proper hepatic artery via intact pancreaticoduodenal arcades.

This procedure has been indicated in locally advanced lesions involving the celiac axis without invasion of the pancreatic head, proper hepatic or superior mesenteric arteries, or abnormal SMA anatomy. The celiac artery must be resectable at its aortic origin and the common hepatic artery must be resected proximal to the take-off of the GDA. Of utmost importance, there should be clear pulsations and arterial Doppler signals at the proper hepatic artery after occlusion of the common hepatic artery to be confident that resection of the $\mathrm{CHA}$ would not result in potentially catastrophic hepatic ischemia.

The most dreaded complications of this procedure are ischemic in nature. Hepatic ischemia can develop related to insufficient flow via the proper hepatic artery (PHA) after ligation of the CHA. Additionally, gastric ischemia can occur due to poor collateral flow in the setting of disruption of the left and right gastroepiploic arteries. Yamamoto et al. report an incidence of ischemic complications in up to $92 \%$ with this procedure. In an effort to decrease the rates of ischemic complications, strategies such as preoperative coil embolization and vascular reconstruction have been employed (Yamamoto 2012). Hirano et al. performed preoperative embolization of the $\mathrm{CHA}$ and $\mathrm{CA}$ to promote collateralization to the hepatobiliary system via the pancreaticoduodenal arcade and stomach via right gastric, right gastroepipoloic, and the left phrenic arteries, respectively (Hirano 2007). Intraoperatively, if hepatic inflow appears to be insufficient as established via palpation, Doppler ultrasonography (Hirano 2007), hepatic vein oxygen saturation (Miyakawa 2002), fluorescein staining (Gagandeep 2006), or pre- 
and post-clamping common hepatic arterial stump pressures (Mittal 2015), then arterial reconstruction is necessary. Various approaches to vascular reconstruction have been described (Table 1).

Herein, we describe our experience with 3 patients who underwent a modified Appleby procedure with (1) primary end-to-side hepatic artery to aorta bypass, (2) primary CHA to LGA bypass in the setting a replaced left hepatic artery (rLHA), and (3) an aortic to PHA bypass with saphenous vein graft and venous reconstruction (Figure 1).

\section{CASE REPORTS}

\section{Patient \#1}

A 65 year-old male with a history of morbid obesity and a gastric bypass presented with non-radiating abdominal pain for 1 week along with a decrease in appetite and early satiety. His work-up included an endoscopic ultrasound, demonstrating a hypoechoic solid cystic mass in the body of the pancreas. This was subsequently biopsy proven to be pancreatic adenocarcinoma. His Ca 19-9 was elevated to $1440 \mathrm{U} / \mathrm{mL}$. Additional CT imaging revealed a hypodense mass extensively involving the body of the pancreas, encasement of the splenic vein and splenic artery with suspicion that the mass also abuted other branches of the celiac axis. The patient elected to undergo elective resection.

Intraoperatively, thorough exploration of the abdominal cavity revealed no evidence of distant metastases and an intact Roux-en-Y gastric bypass. Exploration of the pancreas revealed a firm mass involving the body of the pancreas with extension into the retroperitoneum which also involved the CHA at its take-off from the celiac axis and the proximal LGA. The gastrocolic ligament was opened and divided along with the short gastric vessels in order to mobilize the 
stomach. The spleen and pancreas were elevated out of the retroperitoneum and divided with careful attention paid to assessing the integrity of the SMA throughout. The splenic vein was divided near its junction with the SMV. In order to complete the resection, the CHA was clamped as close as possible to the aorta. Doppler signals were evaluated at the level of CHA, PHA, GDA, and right gastric artery (RGA) with poor signal noted in the hepatic inflow, confirmed by transducing the pressure within the CHA. Given that the CHA was not contributing to the collateral flow via the RGA supplying the stomach following the patient's gastric bypass, the CHA was reimplanted directly into the aorta. Total operative time was 11 hours with an estimated blood loss was $1500 \mathrm{cc}$. Pathology revealed a G2T4N1 pancreatic ductal adenocarcinoma with negative resection margins. The patient's postoperative course was without complication and he was discharged home on post-operative day 6 . He subsequently completed a course of chemotherapy with gemcitabine, paclitaxel, and 5-fluorouracil in combination with radiation therapy. He remains disease-free at 11 months post-resection.

\section{Patient \#2}

A 57 year-old male presented with lower abdominal cramping, change in bowel habits, and new-onset, rapidly progressing diabetes. CT imaging identified a hypodense mixed cystic and solid mass in the central pancreas involving the distal celiac, splenic, and proximal common hepatic arteries with possible splenic and portal vein thrombosis. At the time of evaluation his Ca 19-9 level was 10,334 units/ml.

He underwent (add number) cycles of neoadjuvant FOLFIRINOX and radiation therapy for 2 months followed by proton therapy for 2 months with a profound response of tumor regression. Repeat CT scan after treatment revealed a stable tumor with continued involvement 
of branches of the celiac artery, but without evidence of dissemination. A PET-CT was without evidence of distant metastasis 4 months prior to his presentation for surgical evaluation. His Ca 19-9 level decremented to 92 units/ml. Upon reevaluation, it was felt that he was a candidate for a modified Appleby operation.

Approximately 7 months from the time of his initial diagnosis, he underwent exploratory laparotomy, open cholecystectomy, distal pancreatectomy and en bloc splenectomy with en bloc resection of the celiac artery with common hepatic artery to left gastric artery bypass.

Intraabdominal examination revealed no metastatic disease and a firm mass in the proximal pancreatic body extending toward the celiac axis.

To begin the resection, the gastrocolic ligament was incised allowing gastric mobilization, preserving the right gastroepiploic arcade. Splenomegaly and left-side portal hypertension were apparent. The SMV and PV were then dissected from the pancreatic neck allowing for division of the pancreatic neck. The pancreatic body/tail and spleen were then mobilized out of the retroperitoneum. During dissection, it was noted that the patient's LGA originated from the left hepatic artery (LHA). When the celiac axis was clamped, poor inflow into the LHA was noted; also, when the LGA was compressed, gastric signals were diminished. Therefore, an end-end bypass of the CHA to LGA was performed, improving perfusion to both the left hepatic lobe and stomach. The celiac artery at the aortic origin was then oversewn and the mass removed en bloc. Total operative time was 9 hours and estimated blood loss was 350 cc. Final pathology revealed a T4 N0/M0 pancreatic ductal adenocarcinoma with 98\% response to neoadjuvant therapy. His post-operative course was uncomplicated and he was discharged to have 6 days post-operatively. He subsequently received adjuvant gemcitabine-based chemotherapy. He ultimately had a prolonged convalescence and was found to have metastatic 
disease 6 months postoperatively. He continues to undergo novel adjuvant therapies and he remains alive __ months post-resection.

\section{Patient \#3}

A 56 year-old female who initially presented with jaundice was found to have an unresectable locally advanced pancreatic ductal adenocarcinoma encasing the celiac axis, SMA and splenic artery. She was treated with biliary stent placement and chemotherapy with 12 cycles of FOLFIRINOX, 12 cycles of gemcitabine and paclitaxel, radiation and concurrent 5fluorouracil. Her Ca 19-9 levels decremented post-treatment from 1500 to $31 \mathrm{U} / \mathrm{mL}$. Repeat imaging revealed a pancreatic neck mass encasing the celiac axis, CHA, RHA, proximal splenic artery, and main portal vein.

Approximately 1 year after her initial diagnosis she elected to proceed with an attempt at resection. Upon exploration of the abdomen, no metastatic disease was found. Her celiac axis and SMV-portal vein (PV) confluence was encased with fibrotic tissue, requiring en bloc celiac axis and partial SMV-portal venous resection via a classic total pancreaticoduodenectomy, with en bloc splenectomy. An aortic to PHA bypass was performed using an autologous saphenous vein graft, while the SMV-PV confluence was reconstructed with a primary veno-venous anastomosis. When preparing the stomach for anastomosis, the antral mucosa was noted to be ischemic so a subtotal distal gastrectomy was performed with a loop gastrojejunal and a downstream Braun entero-enterostomy. Total operative time was 12 hours. Estimated blood loss was $2.5 \mathrm{~L}$. Final pathology revealed a T4N0/M0 invasive pancreatic ductal adenocarcinoma with significant treatment effect. All surgical margins were negative. Her postoperative course was prolonged by ventilator dependence and the development of chylous ascites. She remained 
intubated for several days due to large volume fluid resuscitation. She was maintained on enteric coated aspirin for bypass patency and total parenteral nutrition until her discharge on postoperative day 16. She was readmitted on postoperative day 28 for a right sided empyema and treated with antibiotics, chest tube drainage, and ultimately required VATS decortication. Due to her extensive neoadjuvant treatment, she declined adjuvant therapy. She ultimately developed a pelvic recurrence at 5 months post-opertatively and is currently undergoing chemotherapy with gemcitabine and paclitaxel now 8 months post-resection.

\section{DISCUSSION}

The modified Appleby procedure remains a controversial topic. Meta-analysis of outcomes following arterial resection during pancreatectomy for pancreatic cancer has revealed a significantly increased risk for perioperative mortality and poor survival at 1 and 3 years compared to patients undergoing pancreatectomy without arterial resection (Mollberg 2011). Different series report various average survival. Baumgartner et al reviewed 11 cases of the modified Appleby resection following neoadjuvant therapy, reporting median disease-free survival of 21 weeks and overall survival of only 26 weeks (Baumgartner 2012). Kimura et al. reviewed 11 cases of radical resection reporting average survival post-Appleby of 6.6 months (Kimura 1997). Meta-analyses report median survival from 9.5 to 12 months (Mollberg 2011). Gagandeep et al report disease-free survival of greater than 34 months in their experience (Gagandeep 2006).

One fact that is not debatable is that surgical resection remains the only curative option for pancreatic cancer. In a meta-analysis by Mollenberg et al, it was demonstrated that pancreatectomy with arterial resection was associated with a more favorable survival (Odds ratio $=0.49$ at 1 year and 0.39 at 3 years) when comparing patients whom did not undergo surgical 
resection for locally advanced disease (Mollenberg 2011). As such, the modified Appleby procedure is being employed more commonly in Western countries; giving patients with what were once considered unresectable tumors a chance at cure or more often improved quality of life.

In recent years, the criteria for resectability of pancreatic cancer has been expanded based on the combination of increased experience of pancreaticobiliary surgeons operating in high volume centers in radical and innovative operative approaches as well as perioperative management of patients undergoing pancreatectomy. Additionally, appropriate selection of good risk patients who can tolerate multiple serial aggressive treatments may represent the optimal treatment alterative for patients with locally advanced pancreatic cancer. Patient selection is based on patient factors including age, comorbidities, performance status, the surgeon's assessment of feasibility of procedure based on preoperative evaluation of extent and location of tumor as well as surrounding vasculature for collateral flow and arteries potentially need for bypass, and the patient's strong desire for aggressive therapy.

With the expanded criteria of resectablitiy, more patients with locally advanced disease are undergoing surgical resection. Given extent of local invasion and potential vascular involvement, in addition to the need to resect the celiac axis, vascular bypasses are often needed to "supercharge" the liver and or stomach to provide more inline blood flow and preventing ischemia, which complicates these cases. Several techniques for arterial bypass have been reported to address this problem. The earliest bypasses described include CA to CHA or LGA. Newer approaches include HA to aortic bypasses, but with prosthetic grafts utilized as a conduit. In this study, we identified 11 reports involving 27 patients who underwent distal and total pancreatectomy with arterial reconstruction (AR) after celiac artery resection (TABLE 1). 
The most common AR, performed in 11 patients, was a bypass from the aorta to the CHA using a variety of interposition conduits. In our experience, one patient had a primary side to end aortoCHA bypass, another had a primary end to end bypass of the distal CHA to the LGA in the setting a replaced left hepatic artery (rLHA), while the third patient had an aortic to PHA bypass with saphenous vein graft and venous reconstruction. All of our patients recovered well from their operations without ischemic complications. They are currently 11,10 and 8 months postop, respectively.

\section{CONCLUSIONS}

Patients with locally advanced pancreatic cancer have traditionally been considered unresectable with the only treatment option of chemoradiation; leading to dismal long-term survival. With appropriate patient selection, a multidisciplinary team and an experienced pancreaticobiliary surgeon, more patients are meeting expanded resection criteria after preoperative chemoradiation. In many of these cases, arterial reconstruction is necessary to prevent hepatic and gastric ischemia. Based on our own experience and literature review, modified Appleby procedures with arterial reconstruction provide a potentially curative alternative for many patients, with the hope of improved long-term survival.

[The Discussion needs work!

Paragraph 1 - Set the historical stage, define the topic.

Paragraph 2 - Discuss controversies about the topic, some large series; refer to Table.

Paragraph 3 - Discuss summary data and / or meta-analyses.

Paragraph 4 - Summarize our cases... make a few novel points.

Paragraph 5 - Limitations of our study; why more info is needed.

Paragraph 6 - Role of neoadjuvant or adjuvant therapy.] 


\section{REFERENCES}

Appleby LH. The coelic axis in the expansion of the operation for gastric carcinoma. Cancer. 1953; 6: 704-7

Baumgartner JM, Krasinskas A, Daouadi M, Zureikat A, Marsh W, Lee K, et al. Distal pancreatectomy with en bloc celiac axis resection for locally advanced pancreatic adenocarcinoma following neoadjuvant therapy. J.Gastrointest.Surg. 2012;16:1152-9.

Bockhorn M, Burdelski C, Bogoevski D, Sgourakis G, Yekebas EF, Izbicki JR. Arterial en bloc resection for pancreatic carcinoma. Br.J.Surg. 2011;98:86-92.

Christians KK, Pilgrim CH, Tsai S, Ritch P, George B, Erickson B, et al. Arterial resection at the time of pancreatectomy for cancer. Surgery. 2014;155:919-26.

Egorov VI, Petrov RV, Lozhkin MV, Maynovskaya OA, Starostina NS, Chernaya NR, et al. Liver blood supply after a modified appleby procedure in classical and aberrant arterial anatomy. World J.Gastrointest.Surg. 2013;5:51-61.

Gagandeep S, Artinyan A, Jabbour N, Mateo R, Matsuoka L, Sher L, et al. Extended pancreatectomy with resection of the celiac axis: The modified appleby operation. Am.J.Surg. 2006;192:330-5.

Hirano S, Kondo S, Hara T, Ambo Y, Tanaka E, Shichinohe T, et al. Distal pancreatectomy with en bloc celiac axis resection for locally advanced pancreatic body cancer: Long-term results. Ann.Surg. 2007;246:46-51. 9 
Ielpo B, Ferri V, Caruso R, Duran H, Diaz E, Fabra I, et al. Alternative arterial reconstruction after extended pancreatectomy. case report and some considerations of locally advanced pancreatic cancer. Jop. 2013;14:432-7.

Kimura W, Han I, Furukawa Y, Sunami E, Futakawa N, Inoue T, et al. Appleby operation for carcinoma of the body and tail of the pancreas. Hepatogastroenterology. 1997;44:387-93.

Kondo S, Ambo Y, Katoh H, Hirano S, Tanaka E, Okushiba S, et al. Middle colic arterygastroepiploic artery bypass for compromised collateral flow in distal pancreatectomy with celiac artery resection. Hepatogastroenterology. 2003;50:305-7.

Konishi M, Kinoshita T, Nakagori T, Inoue K, Oda T, Kimata T, et al. Distal pancreatectomy with resection of the celiac axis and reconstruction of the hepatic artery for carcinoma of the body and tail of the pancreas. J.Hepatobiliary.Pancreat.Surg. 2000;7:183-7.

Machado MA, Surjan RC, Nishinari K, Makdissi FF, Machado MC. Iliac-hepatic arterial bypass for compromised collateral flow during modified appleby operation for advanced pancreatic cancer. Eur.J.Surg.Oncol. 2009;35:1124-7.

Makary MA, Fishman EK, Cameron JL. Resection of the celiac axis for invasive pancreatic cancer. J.Gastrointest.Surg. 2005;9:503-7.

Mittal A, de Reuver PR, Shanbhag S, Staerkle RF, Neale M, Thoo C, et al. Distal pancreatectomy, splenectomy, and celiac axis resection (DPS-CAR): Common hepatic arterial stump pressure should determine the need for arterial reconstruction. Surgery. 2015;157:811-7. 
Miyakawa S, Horiguchi A, Hanai T, Mizuno K, Ishihara S, Niwamoto N, et al. Monitoring hepatic venous hemoglobin oxygen saturation during appleby operation for pancreatic cancer. Hepatogastroenterology. 2002;49:817-21.

Mollberg N, Rahbari NN, Koch M, Hartwig W, Hoeger Y, Buchler MW, et al. Arterial resection during pancreatectomy for pancreatic cancer: A systematic review and meta-analysis. Ann.Surg. 2011;254:882-93.

Nimura Y, Hattori T, Miura K, Nakashima N, Hibi M. Resection of advanced pancreatic bodyail carcinoma by Appleby’s operation. Shujutu. 1976; 30: 885-889.

Smoot RL, Donohue JH. Modified appleby procedure for resection of tumors of the pancreatic body and tail with celiac axis involvement. J.Gastrointest.Surg. 2012;16:2167-9.

Suzuki H, Hosouchi Y, Sasaki S, Araki K, Kubo N, Watanabe A, et al. Reconstruction of the hepatic artery with the middle colic artery is feasible in distal pancreatectomy with celiac axis resection: A case report. World J.Gastrointest.Surg. 2013;5:224-8.

Wu X, Tao R, Lei R, Han B, Cheng D, Shen B, et al. Distal pancreatectomy combined with celiac axis resection in treatment of carcinoma of the body/tail of the pancreas: A single-center experience. Ann.Surg.Oncol. 2010;17:1359-66.

Yamamoto Y, Sakamoto Y, Ban D, Shimada K, Esaki M, Nara S, et al. Is celiac axis resection justified for T4 pancreatic body cancer?. Surgery. 2012;151:61-9.

[References need to be properly formatted.] 
TABLE 1: Reported Cases of Vascular Reconstruction During the Modified Appleby Procedure

\begin{tabular}{|c|c|c|c|}
\hline First author (year) & Bypass & Reason for bypass & Conduit \\
\hline $\begin{array}{l}\text { Konishi (1992 - } \\
\text { 1998) }\end{array}$ & CA to $\mathrm{CHA}(\mathrm{n}=2)$ & $\begin{array}{l}\text { Weak pulsatility of } \\
\text { PHA after clamping } \\
\text { CA }\end{array}$ & Splenic artery autograft \\
\hline $\begin{array}{l}\text { Kondo (1997 - } \\
\text { 2001) }\end{array}$ & $\begin{array}{l}\text { L branch of middle } \\
\text { colic artery to GEA ( } \\
=2 \text { ) }\end{array}$ & $\begin{array}{l}\text { Accidental injury to } \\
\text { inferior } \\
\text { pancreaticoduodenal } \\
\text { artery during nerve } \\
\text { sheath dissection and } \\
\text { application of } \\
\text { vascular clamp to } \\
\text { SMA with visual } \\
\text { evidence of gastric } \\
\text { ischemia }\end{array}$ & $\begin{array}{l}\mathrm{N}^{*} \\
\text { *requires microsurgical } \\
\text { technique }\end{array}$ \\
\hline Miyakawa (2002) & CA to $\mathrm{CHA}$ & $\begin{array}{l}\text { Measured hepatic } \\
\text { venous hemoglobin } \\
\text { saturation = } 39 \% \\
\text { after clamping CHA }\end{array}$ & Y (IMV graft) \\
\hline $\begin{array}{l}\text { Gagandeep (2002 - } \\
\text { 2004) }\end{array}$ & Celiac stump to LGA & $\begin{array}{l}\text { Fluorescein evidence } \\
\text { of delayed gastric } \\
\text { perfusion }\end{array}$ & $\mathrm{N}$ \\
\hline
\end{tabular}




\begin{tabular}{|c|c|c|c|}
\hline $\begin{array}{l}\text { Machado (pub. } \\
\text { 2009) }\end{array}$ & L EIA to HA & $\begin{array}{l}\text { Poor pulsatility of } \\
\text { PHA after clamping } \\
\text { CHA }\end{array}$ & Y (Dacron) \\
\hline $\mathrm{Wu}(2003-2008)$ & CA to $\mathrm{CHA}(\mathrm{n}=4)$ & $\begin{array}{l}\text { poor pulsatility of } \\
\text { PHA after clamping } \\
\text { CHA }\end{array}$ & $\begin{array}{l}\text { N* } \\
\text { * end-to-end if tension } \\
\text { free anastomosis was } \\
\text { feasible; if not "SA, IMV } \\
\text { or saphenous vein could } \\
\text { be used for graft" }\end{array}$ \\
\hline $\begin{array}{l}\text { Bockhorn (1994 - } \\
\text { 2007) }\end{array}$ & $\begin{array}{l}\text { 1. Aorta/SMA to } \\
\text { PHA }(n=7) \\
\text { 2. Abberant LHA } \\
\text { to PHA }\end{array}$ & & $\begin{array}{l}\text { 1. Cryopreserved } \\
\text { graft } \\
\text { 2. SVG }\end{array}$ \\
\hline $\begin{array}{l}\text { Baumgartner (pub. } \\
\text { 2012) }\end{array}$ & $\begin{array}{l}\text { Supra-celiac aorta to } \\
\text { CHA }\end{array}$ & Prior ligation of GDA & $\mathrm{Y}$ \\
\hline Ielpo (2013) & Aorta to hepatic artery & $\begin{array}{l}\text { Inadequate hepatic } \\
\text { inflow as determined } \\
\text { by intraoperative } \\
\text { Doppler } \\
\text { ultrasonography }\end{array}$ & Y (Dacron) \\
\hline Suzuki (2013) & $\begin{array}{l}\text { PHA to middle colic } \\
\text { artery }\end{array}$ & $\begin{array}{l}\text { Inability to preserve } \\
\text { GDA due to close } \\
\text { proximity to CA }\end{array}$ & $\mathrm{N}$ \\
\hline
\end{tabular}




\begin{tabular}{|l|l|l|l|}
\hline Christians (2001 - & CA to CHA (n=4) & Default unless & Y (rSVG) \\
& & palpable pulses & appreciated in PHA \\
and RGEA after CA & resection & \\
\hline Mittal (2015) & CA to CHA (n=2) & $>25 \%$ decrease in & Y (SVG, PTFE) \\
& & CHA pressure upon & \\
& & clamping CA & \\
\hline
\end{tabular}

[Define all abbreviations in the Table here.] 


\section{FIGURE 1: Depictions of vascular reconstructions performed during modified Appleby}

\section{procedure}
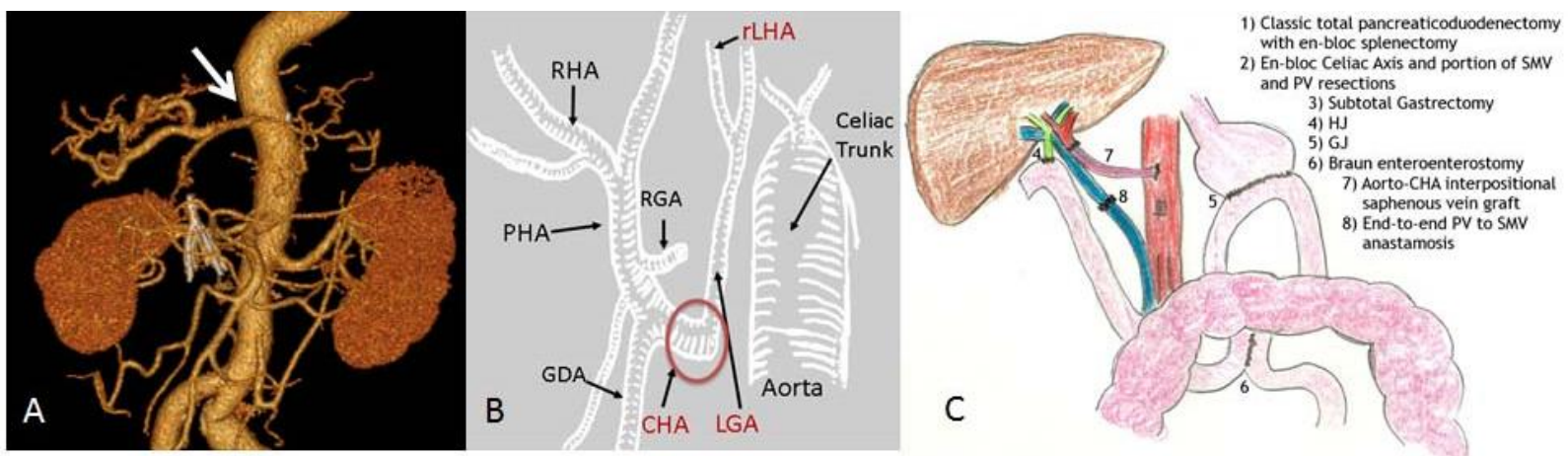

A. Postoperative three-dimensional computed tomography angiogram of Patient \#1 showing the patency of the primary aortic to common hepatic artery bypass (arrow). B. Schematic representation of the postoperative arterial reconstruction of patient \#2 showing a primary end to end bypass of the distal common hepatic artery (CHA) to the left gastric artery (LGA) in the setting of a replaced left hepatic artery (rLHA). C. Schematic representation of the postoperative anatomy of patient \#3 showing classic total pancreatectomy with en bloc splenectomy, celiac axis and SMV-Portal vein resection followed by subtotal gastrectomy, aortic to CHA bypass with RSVG (7) and primary PV to SMV reconstruction (8). 